\title{
REVISIÓN
}

\section{Guía clínica para la resección mucosa endoscópica de lesiones colorrectales no pediculadas}

Eduardo Albéniz', María Pellisé2 ${ }^{2}$ Antonio Z. Gimeno García ${ }^{3}$, Alfredo José Lucendo ${ }^{4}$, Pedro A. Alonso Aguirre ${ }^{5}$, Alberto Herreros de Tejada ${ }^{6}$, Marco Antonio Álvarez ${ }^{7}$, María Fraile ${ }^{8}$, Maite Herráiz Bayod ${ }^{9}$, Leopoldo López Rosés ${ }^{10}$, David Martínez Ares ${ }^{11}$, Akiko Ono ${ }^{12}$, Adolfo Parra Blanco ${ }^{13}$, Eduardo Redondo ${ }^{14}$, Andrés Sánchez Yagüe ${ }^{15}$, Santiago Soto ${ }^{16}$, José Díaz Tasende ${ }^{17}$, Marta Montes Díaz', Manuel Rodríguez Téllez ${ }^{18}$, Orlando García ${ }^{19}$, Alba Zuñiga Ripa', Marta Hernández Conde ${ }^{6}$, Fernando Alberca de las Parras ${ }^{12}$, Carla Gargallo ${ }^{20}$, Esteban Saperas ${ }^{21}$, Miguel Muñoz Navas ${ }^{9}$, Javier Gordillo', Felipe Ramos Zabala ${ }^{22}$, José Manuel Echevarría ${ }^{23}$, Marco Bustamante ${ }^{24}$, Mariano González Haba ${ }^{13}$, Ferrán González Huix ${ }^{25}$, Begoña González Suárez², Juan José Vila Costas', Carlos Guarner Argente ${ }^{26}$, Fernando Múgica ${ }^{27}$, Julyssa Cobián ${ }^{26}$, Joaquín Rodríguez Sánchez ${ }^{28}$, Bartolomé López Viedma ${ }^{27}$, Noel Pin ${ }^{16}$, José Carlos Marín Gabriel ${ }^{17}$, Óscar Nogales ${ }^{29}$, Joaquín de la Peña ${ }^{30}$, Francisco Javier Navajas León ${ }^{31}$, Helena León Brito ${ }^{32}$, David Remedios ${ }^{16}$, José Miguel Estebann33, David Barquero ${ }^{19}$, Juan Gabriel Martínez Cara ${ }^{14}$, Felipe Martínez Alcalá ${ }^{34}$, Ignacio Fernández Urién ${ }^{1}$ y Eduardo Valdivielso5; en nombre del Grupo Español de Resección Endoscópica de la Sociedad Española de Endoscopia Digestiva

${ }^{1}$ Complejo Hospitalario de Navarra. Pamplona. ${ }^{2}$ Hospital Universitari Clínic. Barcelona. ${ }^{3}$ Hospital Universitario de Canarias. San Cristóbal de La Laguna, Santa Cruz de Tenerife. ${ }^{4}$ Hospital General de Tomelloso. Tomelloso, Ciudad Real. ${ }^{5}$ Complejo Hospitalario Universitario de A Coruña. A Coruña. ${ }^{6} \mathrm{Hospital}$ Universitario Puerta de Hierro. Madrid. ${ }^{7}$ Hospital del Mar. Barcelona. ${ }^{8} \mathrm{Hospital}$ San Pedro. Logroño. ${ }^{9} \mathrm{Clínica}$ Universidad de Navarra. Pamplona. ${ }^{10} \mathrm{Hospital}$ Universitario Lucus Augusti. Lugo. ${ }^{11}$ Complejo Hospitalario de Vigo. Vigo. ${ }^{12}$ Hospital Universitario Virgen de la Arrixaca. Murcia. ${ }^{13}$ NIHR Nottingham Digestive Diseases Biomedical Research Unit. Nottingham, Reino Unido. ${ }^{14}$ Hospital Universitario Virgen de las Nieves. Granada. ${ }^{15} \mathrm{Hospital}$ Costa del Sol. Marbella, Málaga. ${ }^{16}$ Complejo Hospitalario de Ourense. Ourense. ${ }^{17}$ Hospital Universitario 12 de Octubre. Madrid. ${ }^{18}$ Hospital Universitario Virgen Macarena. Sevilla. ${ }^{19}$ Hospital Moisés Broggi. Sant Joan Despí, Barcelona. ${ }^{20}$ Hospital Clínico Universitario Lozano Blesa. Zaragoza. ${ }^{21}$ Hospital General de Catalunya. Sant Cugat del Vallés, Barcelona. ${ }^{22}$ Hospital Universitario HM Montepríncipe. Madrid. ${ }^{23}$ EuropaColon España. Madrid. ${ }^{24}$ Hospital Universitari i Politècnic La Fe. Valencia. ${ }^{25} \mathrm{Hospital}$ Universitari Doctor Josep Trueta. Girona. ${ }^{26} \mathrm{Hospital}$ de la Santa Creu i Sant Pau. Barcelona. ${ }^{27} \mathrm{Hospital}$ Universitario Donostia. Donostia-San Sebastián. ${ }^{28} \mathrm{Hospital}$ General de Ciudad Real. Ciudad Real. ${ }^{29} \mathrm{Hospital}$ General Universitario Gregorio Marañón. Madrid. ${ }^{30}$ Hospital de Valdecilla. Santander. ${ }^{31}$ Complejo Hospitalario de Toledo. Toledo. ${ }^{32}$ Hospital Reina Sofía. Tudela, Navarra. ${ }^{33}$ Hospital Universitario Clínico San Carlos. Madrid. ${ }^{34}$ Centro Andaluz de Gastroenterología Integral. Sevilla

Recibido: 29/05/2017 - Aceptado: 07/07/2017

Correspondencia: Maria Pellisé. Servicio de Gastroenterología. Hospital Clínic. C/Villaroel, 170. 08036 Barcelona. e-mail: mpellise@clinic.cat

\section{RESUMEN}

Este documento resume el contenido de la Guía de resección mucosa endoscópica elaborada por el grupo de trabajo de la Sociedad Española de Endoscopia Digestiva (GSEED de Resección Endoscópica) y expone las recomendaciones sobre el manejo endoscópico de las lesiones neoplásicas colorrectales superficiales.

Palabras clave: Resección mucosa endoscópica.

"Esta guía clínica del grupo de trabajo de la Sociedad Española de Endoscopia Digestiva (GSEED de Resección Mucosa) se ha publicado simultáneamente en la Revista Española de Enfermedades Digestivas y en Gastroenterología y Hepatología, debido a su especial interés."
Albéniz E, Pellisé M, Gimeno García AZ, Lucendo AJ, Alonso Aguirre PA, Herreros de Tejada A, Álvarez MA, Fraile M, Herráiz Bayod M, López Rosés L, Martínez Ares D, Ono A, Parra Blanco A, Redondo E, Sánchez Yagüe A, Soto S, Díaz Tasende J, Montes Díaz M, Rodríguez Téllez M, García 0, Zuñiga Ripa A, Hernández Conde M, Alberca de las Parras F, Gargallo C, Saperas E, Muñoz Navas M, Gordillo J, Ramos Zabala F, Echevarría JM, Bustamante M, González Haba M, González Huix F, González Suárez B, Vila Costas JJ, Guarner Argente C, Múgica F, Cobián J, Rodríguez Sánchez J, López Viedma B, Pin N, Marín Gabriel JC, Nogales O, De la Peña J, Navajas León FJ, León Brito H, Remedios D, Esteban JM, Barquero D, Martínez Cara JG, Martínez Alcalá F, Fernández Urién I, Valdivielso E; en nombre del Grupo Español de Resección Endoscópica de la Sociedad Española de Endoscopia Digestiva. Guía clínica para la resección mucosa endoscópica de lesiones colorrectales no pediculadas. Rev Esp Enferm Dig 2018;110(3):179-194.

DOI: $10.17235 /$ reed.2018.5086/2017 


\section{INTRODUCCIÓN}

La resección mucosa endoscópica (RME) es una de las técnicas endoscópicas que han modificado el panorama terapéutico de las lesiones y neoplasias superficiales del tracto gastrointestinal, constituyendo una alternativa eficaz y segura al tratamiento quirúrgico convencional. A pesar de que es un procedimiento que se realiza de forma rutinaria en todas las unidades de endoscopia digestiva, existe gran variabilidad en cuanto a su eficacia y aspectos técnicos. Las resecciones incompletas/recidivas y el sangrado diferido constituyen las principales complicaciones de la RME en el colon. Es preciso estandarizar la técnica para mejorar la tasa de resecciones curativas y minimizar las complicaciones. Desde el grupo español de Resección Endoscópica de la Sociedad Española de Endoscopia Digestiva (GSEED de Resección Endoscópica) se planteó la necesidad de elaborar una guía clínica sobre el tratamiento endoscópico de las lesiones colorrectales.

\section{METODOLOGÍA}

Inicialmente, en junio de 2014, se solicitó a diversos miembros del GSEED de Resección Endoscópica que elaboraran de forma independiente el guión de dicha guía con las preguntas o apartados a desarrollar. Se designó un panel de expertos en RME (Apéndice 1) para configurar cada uno de los apartados de la guía y el contenido principal de los mismos con sus palabras clave correspondientes, entre las que destacan: endoscopic mucosal resection, mucosectomy, polypectomy, endoscopic submucosal dissection, colorectal surgery, indications, contraindications, colorectal cancer, colorectal polyps, granular and non-granular laterally-spreading lesions, quality indicators, antiagregants, anticoagulants, informed consent, patient rights, high-definition colonoscopy, conventional and virtual chromoendoscopy, submucosal invasion, morphologic (Paris, Kudo, Sano, NICE) and histologic (Vienna) classifications, lymph node invasion risk factors, technical aspects of EMR (en-bloc EMR, piecemeal EMR, injection, snare, electrosurgical current, tattoo), bleeding, perforation, post-polypectomy syndrome, complications of sedation, global efficacy, incomplete resection, recurrence, surveillance, surveillance period, learning y competence. En la guía se incluyeron varios participantes con escasos o nulos conocimientos técnicos en RME (metodólogos, médicos residentes, personal de enfermería, asociaciones de pacientes) para asegurar la máxima objetividad de los posicionamientos de la guía. Todos los participantes declararon que no tenían ningún conflicto de interés que interfiriera en la realización de dicha guía.

En su elaboración se siguieron y cumplieron todas las recomendaciones generales del Guidelines International Network (1) (Apéndice 2) y se utilizaron los niveles de evidencia del Scottish Intercollegiate Guidelines Network (SIGN) (2) (Apéndice 3). Se formaron grupos de trabajo de 2-5 personas dirigidas por un coordinador para cada apartado de la guía, que realizaron una revisión independiente de la literatura escrita en inglés en las principales bases de datos disponibles (Cochrane Library, PubMed, MEDLINE, Pubmed Central [PMC], Embase, Scopus); en otras publicaciones secundarias como Bandolier, ACP Journal Club, Clinical Evidence y UpToDate; y en buscadores de internet como TRIP database y SumSearch. Tras dicha revisión de la literatura se realizó un borrador preliminar de la guía que fue discutido y corregido en la reunión de la Sociedad Española de Endoscopia Digestiva SEED en Sevilla en noviembre de 2014.

Los diferentes capítulos fueron revisados por los coordinadores, introduciéndose las modificaciones pertinentes (periodo de enero a junio de 2015). Finalmente, la guía fue sometida a revisión externa por un panel de expertos (periodo de julio a noviembre de 2015). Las nuevas modificaciones fueron revisadas y consensuadas de nuevo por los coordinadores de apartado. El 1 de marzo de 2016 se realizó una reunión de consenso entre los coordinadores generales de la guía, los coordinadores de cada apartado y los revisores externos. Como resultado final se elaboró un documento completo de la guía remitido para edición en formato impreso y digital y que puede consultarse en el siguiente enlace: http://wseed.es/index.php/enlaces/publicaciones/guias-clinicas. El presente documento muestra un resumen de la guía con las recomendaciones obtenidas tras la reunión de consenso.

El objetivo de la Guía es servir como documento de base para los médicos españoles que se dediquen a la endoscopia digestiva. La redacción de la Guía ha sido laboriosa ya que ha buscado la exhaustividad y el consenso y ha implicado a un gran número de profesionales. Es previsible que determinados contenidos puedan quedar obsoletos o faltos de rigor en poco tiempo. Por ello, se considera este documento como un punto de partida y se contempla una actualización en 2021. Las recomendaciones se han basado siempre que ha sido posible en la evidencia científica disponible. Al tratarse de una guía sobre una técnica endoscópica, en muchos puntos la evidencia científica es débil o inexistente $y$, en tal caso, las recomendaciones se han basado en el consenso. Como es obvio, es imprescindible que cada médico considere la aplicación de las recomendaciones en función de sus medios y su experiencia.

\section{INDICACIONES}

Los costes y las complicaciones de la cirugía abierta o laparoscópica de las neoplasias colorrectales precoces son significativamente superiores a los de los tratamientos locales. Considerando que la mayoría de las lesiones colorrectales carecen de potencial invasivo, los tratamientos locales son de elección ya que suelen ser curativos (3-6).

Así pues, las indicaciones de la RME vienen condicionadas por la caracterización histológica, el tamaño de la lesión (hasta $20 \mathrm{~mm}$ se puede plantear una RME en bloque; a partir de $20 \mathrm{~mm}$ habrá que considerar la RME fragmentada [RME-f]) y su localización. Las neoplasias colorrectales precoces sin sospecha de componente invasivo (neoplasia intraepitelial no invasiva, categorías III-IV de la Clasificación de Viena), por su nula posibilidad de presentar metástasis locorregionales o a distancia, pueden ser manejados con tratamiento local. En estos casos, al no ser necesario satisfacer los criterios de resección oncológicos, no es imprescindible realizar una resección en bloque de la lesión. Por otro lado, cuando la lesión neoplásica precoz presenta un componente invasivo que afecta la submucosa o más allá, la resección endoscópica no permite resecar las adenopa- 
tías locorregionales y resulta insuficiente desde un punto de vista oncológico. El colon, a diferencia del resto del tubo digestivo, presenta la peculiaridad de que los vasos linfáticos se sitúan en planos profundos de la submucosa. Esto conlleva que los cánceres colorrectales invasivos que afectan a la capa más superficial de la submucosa $(<1$ $\mathrm{mm}$ ) y presentan criterios histológicos de buen pronóstico (alto grado de diferenciación (G1), no invasión linfática ni vascular, no tumor budding) se asocian a una tasa de afectación ganglionar insignificante y pueden ser tratados de la misma forma que las lesiones sin componente invasivo. En este grupo de lesiones, es preferible la obtención de una pieza única y por lo tanto, la extirpación en bloque de la lesión que permita una adecuada valoración histológica de la misma (5-7).

Las lesiones polipoides (pediculadas [0-Ip]) son tratadas mediante polipectomía convencional. Las lesiones con componente plano o sésil mayores de $10 \mathrm{~mm}$ (0-Ila y 0 -Is) serán tratadas de forma habitual mediante RME. Otras alternativas terapéuticas locales para las lesiones no pediculadas (LCRNP) son: la disección submucosa endoscópica (DSE) y, en el recto, las técnicas de cirugía endoscópica transanales (transanal minimally invasive surgery [TAMIS] o transanal endoscopic microsurgery [TEM]). La DSE consigue tasas de resección en bloque superiores a la RME, por lo que sería preferible, especialmente, cuando sea necesario obtener la lesión en una pieza única completa. Sin embargo, la DSE colorrectal es un procedimiento técnicamente exigente, por lo que en estos momentos, en Europa, se recomienda su realización en el seno de estudios clínicos y solo si se cumplen los requisitos previos de resultados en términos de éxito y seguridad equiparables a las series de referencia publicadas. Posiblemente en los próximos años las indicaciones para DSE en el colon irán en aumento, pero en estos momentos es una técnica limitada a pocos centros con suficiente experiencia (ver guía de DSE de la SEED). En lesiones rectales de gran tamaño sin componente invasivo, la RME y la cirugía endoscópica transanal muestran resultados equiparables en eficacia y seguridad, por lo que la elección de una u otra dependerá de factores como la experiencia local en las técnicas. En lesiones rectales con riesgo de invasión submucosa, DSE y cirugía endoscópica transanal obtienen resultados clínicos semejantes, siendo su elección de forma similar condicionada por la experiencia local en cada centro $(8,9)$.

La correcta descripción de la lesión es el primer punto a tener en cuenta a la hora de indicar una RME, ya que el aspecto de la lesión es de gran ayuda para predecir la presencia de cáncer invasivo (5). Aunque esto se va a exponer en detalle en un apartado subsiguiente, exponemos aquí las definiciones básicas:

- LCRNP: lesión colorrectal no pediculada es toda aquella que es sésil o plana y no presenta pedículo. En la clasificación de París corresponderían a los tipos 0-Is y 0-II (apartado "Identificación y caracterización de lesiones"). Estas lesiones representan un 13-29\% de las neoplasias precoces en Occidente, con una prevalencia similar a la descrita en Japón.

- Lesión de extensión lateral (LST o laterally spreading tumor) (apartado "Identificación y caracterización de lesiones"): lesiones de crecimiento predominantemente en extensión superficial y menor de forma exofítica, sésiles, planas o levemente deprimidas (clasificación de París 0-II, 0-II+0-Is, 0-Is+0-II), con un tamaño superior a los $10 \mathrm{~mm}$. Cuando estas lesiones presentan una superficie granular, se denominan LST granulares (LST-G). A su vez, las LST-G se subdividen en dos tipos: LST-G homogéneas, con superficie granular homogénea y nódulos menores de $3 \mathrm{~mm}$ (París 0-Ila); LST-G nodulares mixtas, con superficie granular pero que presenta algún nódulo de mayor tamaño, o alguna zona sésil (París $0-I I a+I s / 0-I s+0-I I a)$. Cuando la LST presenta una superficie regular y lisa, se denominan LST no granulares (LST-NG), que se subdividen en planas elevadas (París 0-Ila) y pseudodeprimidas (París 0-Ilc, combinado o no con 0lla o 0llb).

Más del $80 \%$ de las LST son de tipo LST-G homogénea y mixta. Solo un $15-20 \%$ se correspondería a lesiones LSTNG. En cuanto a la prevalencia de carcinoma invasivo, se estima que hasta el $98 \%$ de la lesiones LST-G homogéneas carecen de componente invasivo, por lo que son subsidiarias de RME en bloque o fragmentada. Las LST-G nodulares mixtas pueden presentar carcinoma invasivo hasta en un $13 \%$ de los casos y este prácticamente siempre asienta en el nódulo dominante, por lo que se recomienda la RME en bloque de los nódulos de mayor tamaño. Las LST-NG presentan componente invasivo hasta en un $12 \%$ de los casos, pero cuando existe un componente pseudodeprimido (París $0-I l a+(I c)$, este puede llegar a ser del $42 \%$. Estas características hacen necesario considerar técnicas de resección en bloque para las LST-NG, de forma que se pueda realizar una adecuada evaluación histológica $(5,10)$.

La evidencia científica disponible en relación a las lesiones neoplásicas colorrectales está basada en los adenomas como lesión precursora del cáncer colorrectal (CCR). Sin embargo, se estima que un $20-30 \%$ de los CCRs se originan en lesiones serradas (LS). Series largas recientes muestran que las LS pueden representar hasta el $16 \%$ de todas las LCRNP remitidas para tratamiento endoscópico. Estas lesiones suelen presentar un aspecto liso y no granular que podría confundirse con el clásico LST-NG. Sin embargo, su pronóstico es mucho más favorable y pueden ser tratadas con RME-f a pesar de presentar un gran tamaño, pues el riesgo de neoplasia invasiva es excepcional. Esta consideración debe tenerse en cuenta a lo largo de todo el documento (11-13).

Los tumores carcinoides rectales mayores de 15-20 mm son subsidiarios de tratamiento quirúrgico, mientras que para los menores de $10 \mathrm{~mm}$ la RME se considera el tratamiento de primera línea, ya sea en su variante de ligadura con banda o con incisión circunferencial previa. En los tumores carcinoides rectales mayores de $10 \mathrm{~mm}$ y menores de 15-20 $\mathrm{mm}$ podría considerarse la DSE (14).

En caso de lesiones situadas en localizaciones difíciles para su resección, como junto a divertículos, válvula ileocecal, orificio apendicular o canal anal, puede valorarse su remisión a centros de referencia (15).

En la tabla 1 se expone el consenso obtenido en el seno del GSEED de Resección Endoscópica sobre la estrategia terapéutica recomendada para el manejo de las neoplasias precoces colorrectales en función del subtipo morfológico y tamaño. 
Tabla 1. Consenso sobre la estrategia terapéutica recomendada para el manejo de las neoplasias precoces colorrectales en función del subtipo morfológico y tamaño

\begin{tabular}{|c|c|c|}
\hline \multirow{2}{*}{ Subtipo morfológico } & \multicolumn{2}{|c|}{ Estrategia } \\
\hline & Tamaño $\leq \mathbf{2 0 - 3 0 ~ m m ~}$ & Tamaño > 20-30 mm \\
\hline LST-G homogéneo & RME & $\begin{array}{l}\text { RME (DSE en los pocos casos de gran extensión y } \\
\text { sospecha invasión SM profunda, preferiblemente en } \\
\text { centros con experiencia) }\end{array}$ \\
\hline LST-G nodular mixto & RME & $\begin{array}{c}\text { Individualizar casos (RME/DSE: si RME, resecar/analizar } \\
\text { nódulos grandes por separado) }\end{array}$ \\
\hline \multirow{2}{*}{ LST-NG plano elevado } & RME & Individualizar casos (RME/DSE) \\
\hline & \multicolumn{2}{|c|}{ Lesión sugestiva de serrada: la RME-F es en general la técnica de elección } \\
\hline LST-NG pseudodeprimido & RME & DSE \\
\hline Ols & Polipectomía/RME & Individualizar casos (RME/DSE) \\
\hline Non-lifting sign & \multicolumn{2}{|c|}{ Individualizar casos (RME, RME híbrida, RME bajo agua, RME + avulsión o APC, DSE, cirugía) } \\
\hline Olp & Polipectomía & $\begin{array}{l}\text { Polipectomía/RME (pedículos grandes o lesiones } \\
\text { semipediculadas) }\end{array}$ \\
\hline Carcinoides rectales & $\begin{array}{l}<10 \mathrm{~mm} \text { : RME (asistida por capuchón, híbrida) } \\
\text { 10-20 mm: DSE }\end{array}$ & $>20$ mm: cirugía \\
\hline Pólipos menores de 20 mm: & \multicolumn{2}{|c|}{$\begin{array}{c}\text { < } 3 \mathrm{~mm} \text { : pinza de biopsia, asa fría, asa caliente } \\
\text { 4-10 mm: asa fría, asa caliente, RME } \\
>10 \mathrm{~mm} \text { : asa caliente/RME }\end{array}$} \\
\hline
\end{tabular}

LST-G: lesión de extensión lateral granular; LST-NG: lesión de extensión lateral no granular; RME: resección mucosa endoscópica; DSE: disección submucosa endoscópica.

\section{Recomendaciones específicas}

- Los tratamientos locales son preferibles a la resección quirúrgica en los pacientes con neoplasias precoces colorrectales, dados sus menores costes y morbi-mortalidad (nivel de evidencia 2++; grado de recomendación B).

- Se recomienda una adecuada valoración de la morfología, el patrón mucoso superficial y la presencia de signos de invasión submucosa profunda previa a la RME de las lesiones colorrectales no pediculadas (LCRNP) (nivel de evidencia 2++; grado de recomendación B).

- De forma general, la RME es una técnica indicada para todas las LCRNP sin sospecha de invasión submucosa profunda y sin límite de tamaño (nivel de evidencia $1+;$ grado de recomendación $A$ ).

- Las LST-G homogéneas constituyen una adecuada indicación de RME, en bloque y fragmentada (RME-f), independientemente de su tamaño (nivel de evidencia $2+$; grado de recomendación $C$ ).

- En las LST-G mixtas en la RME se debe realizar una extirpación en bloque de los nódulos de mayor tamaño (nivel de evidencia 2+; grado de recomendación C).

- Las LST-NG pseudodeprimidas $>20 \mathrm{~mm}$ tienen un riesgo significativo de presentar focos de invasión submucosa, por lo que deben considerarse técnicas alternativas para lograr una resección en bloque (DSE, cirugía) (nivel de evidencia 2+; grado de recomendación C).

- Las LST-NG superiores a $20 \mathrm{~mm}$ deberían ser remitidas a centros con experiencia para su resección endoscópica en bloque, idealmente mediante DSE o técnica quirúrgica alternativa (nivel de evidencia 4; grado de recomendación $D$ ).

- La RME-f es una técnica segura para el tratamiento de las lesiones serradas, incluso en lesiones $>20 \mathrm{~mm}$ (nivel de evidencia 2+; grado de recomendación C).

- La RME debe considerarse como tratamiento de primera línea para los tumores carcinoides rectales $<10$ $\mathrm{mm}$, ya sea en su variante de ligadura con banda o realizando incisión circunferencial previa (DSE-híbrida) (nivel de evidencia 2++; grado de recomendación B).

- Las RME de lesiones en localizaciones difíciles (divertículos, válvula ileocecal u orificio apendicular y canal anal) deberían remitirse a centros de referencia. (nivel de evidencia 4; grado de recomendación $D$ ).

\section{REQUISITOSY PREPARACIÓN DEL PACIENTE}

Se recomienda conocer diversos datos de la historia clínica del paciente antes de la realización de una RME, entre los que destacan: enfermedades transmisibles; comorbilidades importantes como hepatopatía, nefropatía o cardiopatía; la clasificación de riesgo para la anestesia (ASA); reacciones adversas a medicamentos, látex o níquel; consumo de tóxicos; posibilidad de embarazo; tiempo desde la última ingesta y características de la misma; cirugías y endoscopias previas (16).

A día de hoy no está recomendada la realización rutinaria de analítica de sangre, electrocardiograma o radiografía de tórax previamente a la RME (17). 
El electrocauterio utilizado en la RME puede interferir con los marcapasos. En los dispositivos más recientes no es necesario ningún cuidado previo a la RME, pero en los dispositivos más antiguos puede ser beneficiosa la reprogramación a su modo asíncrono en los pacientes que sean dependientes del mismo y en las situaciones en las que se prevea un electrocauterio prolongado. En pacientes portadores de un desfibrilador automático implantable (DAI), es recomendable la consulta con un cardiólogo especialista en arritmias cardiacas antes de la RME, para valorar la desactivación de la función de detección de taquiarritimias del dispositivo DAl y su posterior reprogramación. Hoy en día, el empleo de imanes por parte de cardiólogos y/o anestesistas puede evitar esta desactivación (18).

La aparición de infecciones a distancia clínicamente relevantes en relación con procedimientos endoscópicos es anecdótica, por lo que no se recomienda profilaxis antibiótica para la prevención de endocarditis infecciosa y tampoco en pacientes portadores de prótesis ortopédicas (19).

Tanto el tratamiento antiagregante como el anticoagulante aumentan el riesgo de sangrado, inmediato o diferido, tras la RME. Es recomendable por tanto la suspensión del tratamiento anticoagulante previa a la resección de lesiones mayores de $10 \mathrm{~mm}$. En pacientes de alto riesgo tromboembólico puede realizarse una terapia puente con heparinas de bajo peso molecular. En cuanto al AAS, en sujetos con bajo riesgo trombótico podría plantearse su suspensión previa a la realización de la RME. En cuanto a las tienopiridinas (clopidogrel, prasugrel, ticagrelor, ticlopidina), se recomienda su suspensión 5-7 días antes de la RME. En caso de doble antiagregación, se recomienda suspender las tienopiridinas y mantener el tratamiento con AAS y, si el elevado riesgo tromboembólico no lo permitiera, se recomienda demorar la RME hasta que sea posible la suspensión de las tienopiridinas $(20,21)$.

A continuación, se exponen las principales recomendaciones respecto a los requisitos previos del paciente para poder ser sometido a RME.

\section{Recomendaciones específicas}

- No se recomienda la realización de analítica de sangre, electrocardiograma o radiografía de tórax previos a una RME en ausencia de clínica o antecedentes personales específicos (nivel de evidencia 3; grado de recomendación $D)$.

- En el caso de marcapasos antiguos, se recomienda la reprogramación del dispositivo a su modo asíncrono en los pacientes que no disponen de ritmo propio (paciente dependiente) en los que se prevé un electrocauterio prolongado. Los dispositivos empleados en la actualidad no requieren ningún cuidado específico (nivel de evidencia 4; grado de recomendación D).

- Es recomendable la consulta con un cardiólogo especialista en arritmias cardiacas que esté formado para el control de dispositivos DAI. Dicho especialista deberá ser el responsable de la desactivación de la función de detección de taquiarritmias del dipositivo DAl, al ser beneficioso, y su reprogramación posterior. En la actualidad la utilización de imanes por el cardiólogo o el propio anestesista puede evitar dicha desactivación (nivel de evidencia 3; grado de recomendación $D)$.

- No se recomienda profilaxis antibiótica para la prevención de endocarditis infecciosa en ningún paciente que vaya a ser sometido a la realización de una $\mathrm{RME}$, independientemente de sus factores de riesgo cardiológico (nivel de evidencia 2+; grado de recomendación D).

- No se recomienda profilaxis antibiótica en pacientes portadores de prótesis ortopédicas que vayan a ser sometidos a la realización de una RME (nivel de evidencia 3; grado de recomendación $D$ ).

- El tratamiento anticoagulante incrementa de forma significativa el riesgo de sangrado, por lo que se recomienda su suspensión antes de la realización de una RME (nivel de evidencia 2+; grado de recomendación C).

- En los pacientes con alto riesgo tromboembólico, se recomienda la terapia puente con heparinas de bajo peso molecular (nivel de evidencia 2+; grado de recomendación C).

- Se puede realizar una RME en pacientes en tratamiento con ácido acetil salicílico a dosis $\leq 300 \mathrm{mg} / \mathrm{día}$. Sin embargo, dado que el ácido acetil salicílico aumenta el riesgo de sangrado, en aquellos pacientes con bajo riesgo de fenómenos trombóticos se recomienda su supresión antes de la realización del procedimiento (nivel de evidencia 3; grado de recomendación D).

- Dado que la RME es un procedimiento de alto riesgo hemorrágico, se recomienda suspender las tienopiridininas (clopidogrel, prasugrel, ticagrelor, ticlopidina) 5-7 días antes de su realización (nivel de evidencia 2+; grado de recomendación C).

- En pacientes tratados con doble antiagregación, se aconseja suspender tienopiridinas 5-7 días antes del procedimiento y mantener el tratamiento con ácido acetil salicílico $\leq 300 \mathrm{mg}$. Si el paciente presenta alto riesgo tromboembólico, debe valorarse diferir el procedimiento hasta que sea prudente suspender las tienopiridinas (nivel de evidencia 2+; grado de recomendación C).

\section{INFORMACIÓN AL PACIENTE}

Al paciente se le debe proporcionar la información necesaria para el cuidado de su propia salud de una manera clara, precisa y suficiente. Es necesario prestar un consentimiento informado por escrito en procedimientos diagnósticos y terapéuticos invasivos. El consentimiento debe incluir unos apartados mínimos entre los que se incluyen el nombre, la descripción y los objetivos del procedimiento, así como los riesgos generales y específicos, los beneficios esperados y las alternativas terapéuticas. Por supuesto, son necesarios la identificación y firma del paciente o representante legal y del médico, así como la fecha y la información del derecho a aceptar o rehusar lo que se le propone y a retractarse del consentimiento ya otorgado $(22,23)$.

Dado que la limpieza intestinal es fundamental para identificar de forma precisa la lesión y sus márgenes, se debe instruir a los pacientes en el modo de realizarla según las últimas evidencias científicas disponibles. La difusión de testimonios de otros pacientes puede facilitar la comprensión del procedimiento y mitigar los temores del paciente. 


\section{Recomendaciones específicas}

- Puesto que las lesiones susceptibles de RME son identificadas a menudo durante un procedimiento endoscópico diagnóstico, se recomienda incluir en el consentimiento general de la colonoscopia las consideraciones técnicas más importantes de la RME (nivel de evidencia 4; grado de recomendación $D$ ).

- En procedimientos que a criterio del endoscopista se prevea puedan ser de alta complejidad o riesgo de complicaciones, la RME se puede diferir y precisará de un consentimiento informado específico (nivel de evidencia 4; grado de recomendación D).

- Dado que la limpieza intestinal es fundamental para identificar de forma precisa la lesión y sus márgenes, se debe instruir a los pacientes en el modo de realizarla según las últimas evidencias científicas disponibles (nivel de evidencia 4; grado de recomendación D).

- La difusión de testimonios de otros pacientes facilita la comprensión del procedimiento y mitiga los temores del paciente (nivel de evidencia 4; grado de recomendación $D)$.

\section{IDENTIFICACIÓN Y CARACTERIZACIÓN DE LESIONES}

Las LCRNP, especialmente las planas (París 0-Ila/b), pueden pasar desapercibidas en la colonoscopia diagnóstica. Es por tanto esencial realizar la colonoscopia bajo los estándares de calidad establecidos por las sociedades científicas. Esto implica una preparación colónica adecuada, una colonoscopia completa, un examen escrupuloso de toda la mucosa colónica que incluya un tiempo de retirada apropiado, una correcta técnica de inspección (es aconsejable la medición de la lesión con las pinzas de biopsia o el asa abiertas) y el uso de las técnicas auxiliares de cromoendoscopia cuando sea menester. En concreto, para las lesiones planas, es importante que el endoscopista tenga experiencia en la detección de lesiones sutiles alertadas por un mínimo cambio en la coloración de la mucosa, depósito de restos fecales o moco, desaparición del patrón vascular, y cambio en la rugosidad de la mucosa. La utilización de endoscopia de alta definición y la aplicación de cromoendoscopia (convencional u electrónica) pueden ser de gran ayuda para poner de manifiesto la lesión y para delimitar correctamente sus márgenes. Por ello, es recomendable que los endoscopistas implicados en la técnica de RME cumplan con los estándares más altos de los criterios de calidad de la colonoscopia y estén totalmente familiarizados con las técnicas de cromoendoscopia (24).

En sujetos de riesgo medio se recomienda la tinción selectiva con índigo carmín para la evaluación de lesiones detectadas con visión convencional, pero no se recomienda la realización de pancromoendoscopia (25).

Por otro lado, como ya se ha comentado en el apartado de "Indicaciones", es imprescindible una correcta caracterización de la lesión para poder planear el tratamiento más adecuado. La clasificación de París modificada (26) y la de las $\operatorname{LST}(10,27)$, serán las empleadas para la caracterización morfológica de las lesiones. El aspecto morfológico se correlaciona de manera clara con la posibilidad de presentar componente invasivo. A esto se añade la caracterización de la lesión en función del patrón mucoso y de otros aspectos morfológicos de la lesión. De forma general, la presencia de zonas induradas, convergencia de pliegues, úlceras, friabilidad, neovascularización, aspecto en columna, zonas de retracción, "piel de pollo" alrededor de la lesión y áreas deprimidas sugiere altamente presencia de componente invasivo (28). El conjunto de estas características es superior al signo de non-lifting para predecir la invasión submucosa. Además, el patrón mucoso puede orientar sobre la existencia y profundidad de la infiltración a la submucosa así como diferenciar los pólipos hiperplásicos de los adenomas. La clasificación de Kudo se obtiene tras tinción con colorante y con la utilización de endoscopios de magnificación o, en su defecto, de alta resolución, permite diferenciar 5 patrones diferentes (29). El patrón mucoso de Sano se obtiene con narrow band imaging (NBI) y magnificación (30); La clasificación NICE (NBI International Colorectal Endoscopic Classification) se obtiene con NBI y alta definición (31).

En lesiones subsidiarias de RME, es recomendable evitar la toma de biopsias, la inyección submucosa y el tatuaje próximo a la lesión para evitar la fibrosis submucosa y, de este modo, no dificultar la posterior resección. Se recomienda biopsiar las áreas más sospechosas de malignidad en las lesiones irresecables endoscópicamente, tatuándose en su vecindad.

Finalmente, es imprescindible una correcta interpretación histológica del espécimen. El rol del patólogo es fundamental a la hora de analizar una pieza de resección endoscópica dado que la interpretación histopatológica determinará el manejo terapéutico subsiguiente, así como también los criterios de seguimiento pospolipectomía. La calidad del resultado histológico no solo depende del patólogo sino también del endoscopista y su técnica. Una resección inadecuada dificultará un análisis de la pieza por parte del patólogo, lo cual tendrá implicaciones en el manejo de los pacientes. Siempre que sea posible, el espécimen resecado debe enviarse al patólogo estirado y fijado a una superficie firme de material poroso mediante alfileres, con la superficie mucosa expuesta, estando toda la pieza sumergida en formalina. El patólogo debe prestar especial atención a confirmar si la resección ha sido completa (márgenes laterales y profundos mayores de $1 \mathrm{~mm}$ ), así como a la presencia o ausencia de invasión submucosa. Se debe considerar invasión submucosa superficial cuando el grado de invasión medido desde la muscular de la mucosa es inferior a 1.000 micras. Se recomienda propiciar al máximo la comunicación entre patólogo y endoscopista para poder optimizar al máximo el manejo de estos pacientes (32). En el apéndice 4 , se muestra la clasificación de Viena modificada (33). En el apéndice 5, se muestra un algoritmo para el manejo de los cánceres precoces colorrectales.

El adenocarcinoma colorrectal se define por la OMS como la invasión de células neoplásicas a través de la muscularis mucosae en la submucosa, y se recomienda estratificarlo de acuerdo a la clasificaciónTNM. En caso de cáncer invasivo pT1, debe informarse la presencia o ausencia de criterios de mal pronóstico (grado de diferenciación, profundidad de invasión en la capa submucosa, infiltración linfovascular, presencia de tumor budding). En caso de presentar alguno de estos criterios, el espécimen debe ser valorado por dos patólogos ya que es posible que se requieran tratamientos 
complementarios invasivos (cirugía) (28). Esta recomendación de cirugía adicional en el adenocarcinoma pT1 debería ser determinada por un equipo multidisciplinario.

\section{Recomendaciones específicas}

- En población de riesgo medio, se recomienda la tinción selectiva con índigo carmín para la evaluación de lesiones detectadas bajo visión convencional, pero no el uso de pancromoendoscopia (nivel de evidencia 1+; grado de recomendación $B$ ).

- Se recomienda aplicar técnicas de contraste como la cromoendoscopia de tinción o la óptica y, si está disponible, la magnificación, para estudiar la superficie de las LCRNP (nivel de evidencia 4; grado de recomendación D).

- Se recomienda fotografiar y/o grabar en vídeo todas las LCRNP antes de la resección (nivel de evidencia 4; grado de recomendación $D$ ).

- Se recomienda realizar una estimación del tamaño de las LCRNP con la ayuda de la pinza de biopsia o el asa de polipectomía abiertas (nivel de evidencia 4; grado de recomendación $D$ ).

- Se recomienda usar la clasificación de París para describir la morfología de las lesiones (nivel de evidencia 4; grado de recomendación D).

- Se recomienda describir las características de la superficie de las lesiones colorrectales mediante la clasificación NICE y/o la clasificación de Sano y/o el patrón de criptas de Kudo (nivel de evidencia 4; grado de recomendación $D$ ).

- La presencia de áreas de eritema, consistencia firme, aspecto expansivo, convergencia de pliegues y "piel de pollo" alrededor de la lesión se asocian con mayor riesgo de invasión submucosa (nivel de evidencia 2+; grado de recomendación C).

- La caracterización endoscópica es superior al signo de la no elevación para predecir invasión submucosa (nivel de evidencia 2+; grado de recomendación C).

- En lesiones subsidiarias de resección endoscópica, la toma de biopsias, la inyección submucosa y el tatuaje próximo a la lesión deberían evitarse debido a que pueden provocar fibrosis submucosa y dificultar la futura resección endoscópica (nivel de evidencia 4; grado de recomendación D).

- En lesiones con signos de irresecabilidad endoscópica por sospecha de invasión profunda, la toma de biopsias debe ser dirigida a la zona más sospechosa. Estas lesiones serán tatuadas en vecindad salvo en localizaciones obvias (recto y ciego) (nivel de evidencia 4; grado de recomendación D).

- Se recomienda emplear la clasificación de Viena modificada para el diagnóstico histológico de las neoplasias superficiales del tracto digestivo (nivel de evidencia 4; grado de recomendación D).

- La definición de adenocarcinoma colorrectal de la OMS debe utilizarse como: “invasión de células neoplásicas a través de la muscularis mucosae en la submucosa". Los términos adenocarcinoma intramucoso o carcinoma in situ no deben utilizarse (nivel de evidencia 4; grado de recomendación $D)$.

- El adenocarcinoma se debe estratificar de acuerdo a la clasificación TNM. La versión de clasificación TNM a utilizar debe ser acordada en forma nacional y es- tablecida formalmente por organismos profesionales de ámbito nacional (nivel de evidencia 4; grado de recomendación $D$ ).

- Se recomienda enviar el espécimen resecado esturado y fijado a una superficie firme de material poroso mediante alfileres, con la superficie mucosa expuesta y toda la pieza sumergida en formalina, para asegurar una correcta evaluación de las LCRNP (nivel de evidencia 4; grado de recomendación D).

- El patólogo debe prestar especial atención a poder establecer la resección completa de la lesión neoplásica (márgenes laterales y profundos libres), así como a la presencia o ausencia de invasión submucosa en los especímenes obtenidos (nivel de evidencia 4; grado de recomendación $D$ ).

- En caso de cáncer invasivo pT1, deben reportarse la presencia o ausencia de criterios de mal pronóstico: grado de diferenciación, profundidad de invasión en la capa submucosa, infiltración linfovascular, presencia de tumor budding (nivel de evidencia 1-; grado de recomendación $D$ ).

- Un margen menor o igual a $1 \mathrm{~mm}$ tanto lateral como en profundidad indica margen afecto (nivel de evidencia 3; grado de recomendación D).

- Se debe considerar invasión de la submucosa superficial cuando el grado de invasión medido desde la muscular de la mucosa es inferior a 1.000 micras (nivel de evidencia 3; grado de recomendación D).

- Todo diagnóstico histológico con criterios de mal pronóstico que determine la posibilidad de tratamientos complementarios invasivos (cirugía) debe ser evaluado al menos por dos patólogos (nivel de evidencia 4; grado de recomendación D).

- La recomendación de cirugía adicional en el adenocarcinoma pT1 debería ser determinada por un equipo multidisciplinario (nivel de evidencia 4; grado de recomendación $D)$.

\section{ASPECTOSTÉCNICOS}

La RME es una maniobra rutinaria durante una colonoscopia que presenta diversos grados de dificultad, por lo que la experiencia del endoscopista juega un gran papel en la eficacia del procedimiento. Los factores de la lesión que se asocian a una mayor dificultad técnica son: localización (válvula ileocecal, periapendicular, divertículos y línea dentada), lesión de difícil acceso (por acodamiento o formación de asa), afectación de más del $50 \%$ de la circunferencia, tamaño > $40 \mathrm{~mm}$, morfología LST-NG y ausencia o deficiente elevación tras inyección submucosa (non lifting-sign). La presencia de fibrosis en la submucosa dificulta o incluso puede impedir la inyección de soluciones en esta capa, aumentando de manera secundaria el riesgo de complicaciones y entorpeciendo la resección endoscópica de estas lesiones e incrementando la tasa de resecciones incompletas y la probabilidad de recidiva (34).

La RME se realiza en tres fases: a) maniobras endoscópicas que permitan la colocación de la lesión en la mejor situación para su resección (entre las posiciones horarias 5 y 7) y la elección del material más adecuado (el endoscopista debe estar familiarizado con todo el utillaje disponible); b) los métodos de resección en función del tipo de lesión; y c) la actitud tras la resección (35). 
La insuflación con $\mathrm{CO}_{2}$ en lugar de aire ambiente consigue un mayor perfil de seguridad y mejorar la tolerancia para el paciente. En cuanto al electrocauterio, se recomienda el uso de corrientes controladas por microprocesador (ENDO $\mathrm{CUT}^{\circledR}$ ) por su perfil de eficacia y seguridad. Las corrientes de corte puro se asocian a un mayor riesgo de sangrado inmediato, mientras que las de coagulación pura se asocian a un mayor riesgo de sangrado diferido (36). En lesiones mayores de $20 \mathrm{~mm}$ se recomienda la inyección submucosa con sustancias de baja velocidad de difusión, como por ejemplo glicerina, gelatina succinato, ácido hialurónico o hidroxipropil metilcelulosa, ya que se asocian a una mayor tasa de resecciones completas. Además, es recomendable el empleo de colorantes estériles como índigo carmín o azul de metileno en la dilución de la inyección submucosa, ya que permiten una mejor delimitación de los márgenes de la lesión y del plano de corte $(37,38)$.

En lesiones mayores de $20-30 \mathrm{~mm}$ no se recomienda la RME en bloque por el mayor riesgo de perforación y de resecciones incompletas (39). Es importante completar la RME en una sola sesión, ya que el empleo de más de una sesión se asocia a una mayor tasa de resecciones incompletas. La resección debe completarse con el asa de diatermia, reservando las técnicas ablativas para islotes mucosos residuales. La escara post-RME debe revisarse de forma meticulosa para evaluar los márgenes y la integridad de la capa muscular (40). La RME mediante técnica de inmersión es una alternativa que puede ser de utilidad en los casos de lesiones residuales sobre escaras de resecciones previas (41). La inyección de anestésicos locales mejora la tolerancia en resecciones cercanas a la línea dentada (42). En lesiones situadas tras pliegues o en la parte intravalvular de la válvula ileocecal es útil el uso de capuchones o endoscopios flexibles que permitan la retroflexión (39). El empleo de bandas en el colon no se recomienda por el elevado riesgo de perforación. La hemorragia intraprocedimiento puede controlarse con APC, fórceps de coagulación o con la punta del asa de polipectomía en modo de coagulación "soft". No es recomendable utilizar los clips hemostáticos hasta finalizar la resección, siempre que sea posible (43).

La técnica recomendada para el tatuaje de la zona consiste en introducir la aguja de forma tangencial a la mucosa e inyectar en primer lugar suero fisiológico para crear un habón submucoso, donde posteriormente se inyectará carbón y/o tinta china (44).

A pesar de ser una técnica que se realiza de forma rutinaria y con gran frecuencia desde hace al menos una década, muchos de los aspectos técnicos no han sido evaluados científicamente y se aplican de forma empírica. A continuación, exponemos los puntos clave para la correcta realización de la RME, ahondando en aquellos en los que existe evidencia científica y en aquellos que, por consenso, se consideran importantes.

\section{Recomendaciones específicas}

- La experiencia del endoscopista tiene un impacto muy significativo en la eficacia del procedimiento, incluyendo el riesgo de complicaciones, la tasa de resección en bloque y la tasa de resección completa (nivel de evidencia 4; grado de recomendación $D$ ).
- El endoscopista debe estar familiarizado con todo el utillaje disponible y necesario para la realización de RME (nivel de evidencia 4; grado de recomendación $D)$.

- Se recomienda colocar el pólipo entre las posiciones horarias 5 y 7 ya que es donde se encuentra habitualmente el canal operativo del colonoscopio (nivel de evidencia 2+; grado de recomendación D).

- Se recomienda la insuflación de $\mathrm{CO}_{2}$ en lugar de aire ambiente ya que mejora la tolerancia y el perfil de seguridad para el paciente (nivel de evidencia 2+; grado de recomendación $D$ ).

- Se recomienda la utilización de corrientes controladas por microprocesador (tipo ENDO CUT ${ }^{\circledR}$ ) por su perfil de seguridad y eficacia. En este mismo sentido, se recomienda evitar corrientes de corte puro por el mayor riesgo de sangrado inmediato y la corriente de coagulación pura por el de sangrado diferido (nivel de evidencia 1+; grado de recomendación B).

- Para LCRNP mayores de $20 \mathrm{~mm}$, se recomienda el uso de sustancias de inyección con baja velocidad de difusión en la submucosa (glicerina, ácido hialurónico, hidroxipropil metilcelulosa, succinato de gelatina) ya que logran una tasa de resecciones completas más elevada (nivel de evidencia 1+; grado de recomendación A).

- Se recomienda la utilización de colorantes estériles (índigo de carmín, azul de metileno) en la solución de inyección submucosa para delimitar mejor los márgenes y el plano de corte (nivel de evidencia 4; grado de recomendación $D$ ).

- No se recomienda la RME en bloque en lesiones mayores de 20-30 $\mathrm{mm}$ por un mayor riesgo de perforación o resección incompleta (nivel de evidencia 2++; grado de recomendación $B$ ).

- Se recomienda completar la resección en una sola sesión ya que las resecciones en varias sesiones se han asociado a un mayor riesgo de fracaso terapéutico endoscópico (nivel de evidencia 2+; grado de recomendación $\mathrm{C})$.

- Se recomienda completar la resección con el asa de diatermia y reservar las técnicas ablativas para pequeños islotes de lesión residual (nivel de evidencia 2+; grado de recomendación C).

- Se recomienda revisar la escara post-RME de forma meticulosa, para evaluar los márgenes de resección y la integridad de la capa muscular (nivel de evidencia 3; grado de recomendación D).

- La RME con bandas en el colon no se recomienda al no considerarse segura, salvo en recto, por el riesgo de perforación (nivel de evidencia 1-; grado de recomendación $B$ ).

- Se recomienda valorar la RME mediante técnica de inmersión para la resección de lesiones residuales sobre cicatrices de resección previa (nivel de evidencia 2++; grado de recomendación C).

- Se recomienda la inyección de anestésicos locales en la resección de lesiones en contacto con la línea dentada ya que mejora la tolerancia (nivel de evidencia 3; grado de recomendación $D)$.

- Para controlar la hemorragia intraprocedimiento se recomienda el uso de APC, el fórceps hemostático o la punta del asa de polipectomía en modo de coagulación "soft" (nivel de evidencia 3; grado de recomendación D). 
- Se recomienda el uso de un capuchón o de un endoscopio flexible que permita la retroflexión para resecar lesiones tras ángulos, pliegues o en la parte intravalvular de la válvula ileocecal (nivel de evidencia 4; grado de recomendación D).

- La técnica recomendada para el tatuaje con carbón biocompatible y/o tinta china consiste en introducir la aguja tangencial a la mucosa en la submucosa e inyectar previamente suero fisiológico con el fin de hacer un habón submucoso para posteriormente introducir el carbón y/o la tinta china (nivel de evidencia 4; grado de recomendación $D$ ).

\section{COMPLICACIONES INTRA Y POSPROCEDIMIENTO}

Las complicaciones más importantes de la RME son la hemorragia y la perforación. Pueden ser tratadas y resueltas con métodos endoscópicos en la mayor parte de las ocasiones, si bien pueden precisar cirugía, especialmente tras una perforación. Por otra parte, pueden presentarse complicaciones derivadas de la propia colonoscopia, la preparación intestinal previa, la sedación y la suspensión o sustitución del tratamiento antiagregante o anticoagulante en aquellos pacientes que lo tomen. La ausencia de un criterio homogéneo a la hora de valorar los eventos y, por tanto, de considerarlos como complicación o no es probablemente una de las causas de la amplia variabilidad en la incidencia de complicaciones reportada en la literatura. Además, existen pocos estudios que recojan de forma prospectiva todos los eventos posteriores a la RME, por lo que probablemente las complicaciones menores estén infraestimadas.

La hemorragia es la complicación más frecuente y suele presentarse en las primeras 48 horas, aunque en ocasiones aparece hasta 14 días después del procedimiento. La incidencia de la hemorragia intraprocedimiento oscila entre el $3,4 \%$ y el $24 \%$; la de la hemorragia inmediata, entre el $1 \%$ y el $11 \%$; y la diferida, entre el $0 \%$ y el $9,7 \%$. En la mayor parte de los casos se autolimita sin requerir ningún tratamiento y la mortalidad por esta complicación se puede considerar prácticamente nula. Los factores predisponentes de hemorragia post-RME son: la localización proximal, el tamaño grande de la lesión, la hemorragia intraprocedimiento, el uso de unidades electroquirúrgicas no controladas por microprocesador, la edad avanzada del paciente, la hipertensión arterial y el tratamiento con ácido acetil salicílico o anticoagulantes (45-47). El empleo de adrenalina diluida en la inyección submucosa se asocia a una reducción en la incidencia de la hemorragia precoz, pero no afecta a la incidencia de la hemorragia diferida (48). La coagulación de los vasos visibles con argón o fórceps de coagulación tampoco reduce la incidencia de hemorragia post-RME en colon y recto (49). Tampoco existe evidencia científica que recomiende el cierre sistemático de las escaras con clips hemostáticos para prevenir la hemorragia post-RME, pero el cierre de los defectos mucosos con clips tras la RME de grandes lesiones colónicas sí se asocia a un menor riesgo de sangrado diferido (47).

La tasa de perforación, considerada tradicionalmente un estándar de calidad de la colonoscopia, es del 0,03-0,8\% en procedimientos diagnósticos y del $0,15-3 \%$ en procedimien- tos terapéuticos (50). La RME es una técnica considerada de alto riesgo de perforación por la European Society of Gastrointestinal Endoscopy (ESGE), de forma que se han comunicado tasas de perforación menores del $5 \%$ en caso de grandes lesiones colónicas $(51,52)$. Los factores de riesgo de perforación tras RME incluyen el tamaño grande de la lesión y la localización en colon derecho. Otras características como edad avanzada, comorbilidad, sexo femenino, enfermedad inflamatoria intestinal, cirugías abdominales previas, indicación de colonoscopia por obstrucción, morfología sésil (0-Is), fibrosis o invasión en profundidad de la lesión, mala preparación e inexperiencia del endoscopista también se han descrito como factores de riesgo de perforación en la colonoscopia (53). El diagnóstico precoz es crucial en el manejo de la perforación, determinando la estrategia terapéutica y el pronóstico del paciente. Se detecta inmediatamente en casi un tercio de los casos y en el resto, entre uno y dos días tras el procedimiento. Raramente se observan casos de perforación diferida entre los tres y los 14 días. Tras la RME, es fundamental la inspección del defecto mucoso y descartar la existencia del denominado signo de la diana (target sign). Dicho signo consiste en la visualización de tejido de la muscular en la base de la lesión resecada rodeado de submucosa y se asocia con una alta probabilidad de daño en la capa muscular propia. En estos casos se recomienda cerrar la escara con clips para evitar una perforación tardía y reducir la necesidad de cirugía y mortalidad (3). En caso de perforaciones menores de 2 cm con limpieza aceptable de la luz intestinal, en pacientes estables clínica y analíticamente, el tratamiento podría ser endoscópico. La cirugía se reserva ante el fracaso del tratamiento endoscópico, para las perforaciones mayores de $2 \mathrm{~cm}$, ante la sospecha de sepsis/peritonitis y en aquellos pacientes con diagnóstico tardío de perforación. En caso de neumoperitoneo a tensión, las maniobras de descompresión percutánea mejoran al paciente y sirven de terapia puente hasta el tratamiento definitivo de la perforación. Los antibióticos de amplio espectro deben asociarse tanto al tratamiento endoscópico como al quirúrgico (54).

A continuación, se exponen las medidas preventivas para evitar complicaciones y las recomendaciones para el adecuado manejo de estas.

\section{Recomendaciones específicas}

- La inyección submucosa de adrenalina reduce la incidencia de hemorragia precoz pero no la diferida (nivel de evidencia 1+; grado de recomendación A).

- No se recomienda la coagulación preventiva de los vasos visibles con fórceps o con APC ya que no previene la incidencia de sangrado post-RME en el colon y recto (nivel de evidencia 1++; grado de recomendación A).

- No se recomienda el cierre sistemático con clips de las escaras tras RME para prevenir la hemorragia diferida (nivel de evidencia 2-; grado de recomendación D).

- Se recomienda explorar la escara y ante la detección de daño en la capa muscular cerrar el defecto con clips (nivel de evidencia 3; grado de recomendación D).

- Se recomienda el tratamiento endoscópico en las perforaciones $\leq 2 \mathrm{~cm}$, con limpieza aceptable de la luz intestinal, que suceden en pacientes con estabilidad clínica y analítica (nivel de evidencia 3; grado de recomendación D). 
- Se recomienda el tratamiento quirúrgico ante el fallo del tratamiento endoscópico, perforaciones de gran tamaño, si se sospecha sepsis y/o peritonitis y en aquellos pacientes con diagnóstico tardío de la perforación (nivel de evidencia 3; grado de recomendación D).

- En caso de neumoperitoneo a tensión, se recomiendan maniobras de descompresión percutánea al mejorar la situación clínica del paciente como puente al tratamiento definitivo de la perforación (nivel de evidencia 3; grado de recomendación D).

- Se recomienda el uso de antibióticos de amplio espectro asociado a tratamiento endoscópico o quirúrgico de la perforación (nivel de evidencia 3; grado de recomendación D).

- La sedación no incrementa el riesgo de complicaciones durante la realización de la RME (nivel de evidencia 3; grado de recomendación D).

\section{EFICACIA Y SEGUIMIENTO}

Se estima que el riesgo global de recurrencia tras una RME es del $15 \%$ (IC 95\% 12-19\%), con un 3\% en las resecadas en bloque frente al $20 \%$ en las resecciones fragmentadas. Alrededor del $70 \%$ de las recurrencias son identificables a los tres meses de vigilancia y más del $90 \%$, a los seis meses. Estas recidivas pueden afrontarse nuevamente por medio de un método endoscópico, por lo que se puede concluir que en lesiones sin componente invasivo y que son susceptibles de tratamiento endoscópico, dicho tratamiento es definitivo hasta en el $99 \%$ de los casos tras una media de 1,2 endoscopias, y solo en el 1-4\% de los LCRNP de gran tamaño es necesaria cirugía de rescate. Sin embargo, cuando se tienen en cuenta las resecciones no curativas por invasión en la histología, lesiones sincrónicas, recurrencias no manejables endoscópicamente y complicaciones, las tasas globales de cirugía alcanzan el $9 \%$. Los factores predictores de fracaso terapéutico son: tamaño mayor de $30 \mathrm{~mm}$, resección incompleta inicial, RME fragmentada, morfología 0-Is y 0-Ila+ls, patrones de Kudo III/IV/V, histología adenomatosa versus serrada, displasia de alto grado y carcinoma con invasión submucosa versus displasia de bajo grado $(6,55,56)$.

Se considera que el tratamiento de un pólipo mediante RME ha sido eficaz cuando la lesión está totalmente resecada y sin recidiva tras al menos un primer control. En las RME en bloque con resección completa no es necesaria una colonoscopia precoz para el control de la cicatriz post-RME. En la RME-f, se considera que la primera revisión tras RME completa forma parte del procedimiento terapéutico. Esta revisión debe ir encaminada a explorar de forma detenida la cicatriz post-RME en busca de recidiva. En el caso de las lesiones mayores de $20 \mathrm{~mm}$ se hará a los 3-6 meses. La cromoendoscopia (electrónica y convencional), la endomicroscopía confocal y la toma de biopsias han mostrado ser útiles en la detección de neoplasia residual en cicatrices post-REM (57), y es recomendable su fotografiado. Si se detecta y trata una lesión residual, es recomendable un nuevo control endoscópico a los 6-12 meses, mientras que en ausencia de recidiva el control se recomienda a los 12 meses; esto puede variar según las recomendaciones del propio endoscopista. El tratamiento de lesiones sobre escaras, al ser más complejo, debe ser realizado por un endoscopista experto en RME, y los casos complejos serán evaluados por un comité multidisciplinar para tomar decisiones que serán consensuadas con el paciente (47).

Los individuos con adenomas y lesiones serradas presentan riesgo de presentar lesiones metacrónicas (58), por lo que deben seguir controles con colonoscopia según lo establecido por las guías nacionales y europeas de vigilancia pospolipectomía (59).

\section{Recomendaciones específicas}

- En caso de RME en bloque con resección completa, no es necesario realizar una colonoscopia precoz para control de la cicatriz post-RME. Se realizará la colonoscopia de seguimiento para descartar lesiones metacrónicas siguiendo las guías de vigilancia pospolipectomía (nivel de evidencia 1+; grado de recomendación $B$ ).

- En caso de RME fragmentada de LCRNP mayores de $20 \mathrm{~mm}$ se recomienda control endoscópico en 3-6 meses para descartar recidiva/presencia de tejido residual (nivel de evidencia 1++; grado de recomendación A).

- Cuando se objetive y se trate una recidiva, se recomienda un nuevo control precoz en 6-12 meses (nivel de evidencia 2+; grado de recomendación C).

- En ausencia de recidiva, se recomienda control endoscópico a los 12 meses del control anterior (nivel de evidencia 2+; grado de recomendación C).

- En casos específicos se recomienda seguimiento según la recomendación del endoscopista (nivel de evidencia 4; grado de recomendación D).

- Durante las colonoscopias de seguimiento la escara debe ser identificada, explorada detalladamente y fotografiada. Se recomienda la utilización de técnicas de realce y biopsias, ya que pueden mejorar la detección de neoplasia residual sobre la escara (nivel de evidencia 3; grado de recomendación D).

- Las áreas con sospecha de tejido residual requieren diagnóstico tisular y tratamiento definitivo. En la mayoría de las ocasiones se recomienda tratamiento endoscópico (nivel de evidencia 3; grado de recomendación D).

- El tratamiento de la recurrencia sobre la escara debería ser realizado por un endoscopista experto en RME (nivel de evidencia 3; grado de recomendación D).

- Los casos de recurrencia complejos deberían discutirse en un comité multidisciplinar y las decisiones deberían consensuarse con el paciente (nivel de evidencia 3; grado de recomendación D).

\section{APRENDIZAJEY COMPETENCIA}

La RME es una técnica que se realiza en la mayoría de las unidades de endoscopia de nuestro país y que presenta, en general, una complejidad superior a la polipectomía simple con pinza o asa (fría o de diatermia). Los objetivos del aprendizaje de la RME deben incluir todas las fases del procedimiento, con una evaluación completa de los pacientes: a) reconocer las indicaciones de la técnica; b) alcanzar la adecuada competencia en el procedimiento; c) establecer una adecuada valoración de riesgos/beneficios; y d) conocer el manejo del paciente tras el procedimiento, incluyendo las complicaciones. 
Según la guía europea para la calidad en colonoscopia diagnóstica y de cribado, los médicos habilitados para realizar cribado deben ser capaces de tratar lesiones $<25$ $\mathrm{mm}$ mediante polipectomía convencional y LCRNP $<20$ $\mathrm{mm}$ mediante RME (nivel de capacitación 3). Las LCRNP $>20 \mathrm{~mm}$ o localizadas en posiciones de difícil acceso han de ser tratadas por profesionales con el máximo nivel de capacitación (nivel 4) (60).

La eficacia de la RME, definida por la ausencia de recidiva durante el seguimiento, es superior entre los endoscopistas expertos que entre los inexpertos. Es importante disponer de un volumen adecuado de casos para que los endoscopistas en entrenamiento obtengan la suficiente experiencia. Afortunadamente, la prevalencia de lesiones con indicación de RME en colon es muy elevada, por lo que en nuestro país el volumen de pacientes no debería ser un problema (61).

No existen estudios que evalúen la curva de aprendizaje en RME de colon y recto. Para el Joint Advisory Group (JAG) de Reino Unido, el número aconsejado de procedimientos para la acreditación provisional en colonoscopia es de 200 procedimientos, aunque el número requerido para la acreditación completa es de 300 procedimientos. Para este grupo, el nivel 1 de polipectomía se centra en pólipos de menos de $1 \mathrm{~cm}$ y el nivel 2, en pólipos mayores de $1 \mathrm{~cm}$. Para la RME en especial han elaborado un formulario de evaluación para la valoración de destrezas a través de la observación directa. Los ítems que se incluyen en él son: a) inyección adecuada de la submucosa, empleando una técnica de inyección apropiada; b) realizar la polipectomía solo si la lesión se eleva adecuadamente; c) seleccionar el tamaño apropiado del asa; d) dirigir el asa cuidadosamente sobre la lesión; e) seleccionar adecuadamente entre la resección en bloque o en fragmentos dependiendo del tamaño de la lesión; f) posicionamiento adecuado del asa sobre la lesión cuando se cierra el asa; g) asegurar que la cantidad correcta de tejido es atrapada por el asa; h) separar la lesión cuidadosamente del resto de mucosa sana; i) seleccionar adecuadamente asa fría o diatermia; y j) asegurar una hemostasia adecuada. Estos formularios producen evaluaciones objetivas que resultan en puntuaciones, pero no dan datos de la curva de aprendizaje de la RME. Con algunos cambios de criterios para adaptarse a la realidad del entrenamiento en estas técnicas, el JAG ofrece una certificación completa cuando el endoscopista ha mostrado competencia suficiente en un procedimiento que le permite la práctica independiente (62).

En España, aún carecemos de un sistema de puntuación adaptado o traducido.

\section{Recomendaciones específicas}

- Los endoscopistas noveles en RME deberían ser competentes en colonoscopia diagnóstica y terapéutica básica (polipectomía simple con asa y tratamiento de complicaciones) (nivel de evidencia 4; grado de recomendación D).

- Los endoscopistas noveles en RME deberían saber identificar LCRNP, identificar signos endoscópicos sospechosos de invasión y tener conocimientos de cromoendoscopia (nivel de evidencia 4; grado de recomendación D).

\section{Apéndice 1. Componentes del panel de expertos encargado de realizar la guía}

\section{Coordinadores:}

- Eduardo Albéniz Arbizu. Complejo Hospitalario de Navarra. Pamplona

- María Pellisé Urquiza. Hospital Universitari Clínic. Barcelona

\section{Revisión externa:}

- Antonio Zebenzuy Gimeno García. Hospital Universitario de Canarias. Tenerife

- Alfredo Lucendo Villarín. Hospital General de Tomelloso. Ciudad Real

\section{Introducción:}

- Leopoldo López Rosés. Hospital Xeral. Lugo

\section{Indicaciones:}

- Coordinador: Alberto Herreros de Tejada. Hospital Puerta de Hierro-Majadahonda. Madrid

- Manuel Rodríguez Téllez. Hospital Universitario Virgen Macarena. Sevilla

- Orlando García Bosh. Hospital Moisès Broggi. Barcelona

- Alba Zúñiga Ripa. MIR. Complejo Hospitalario de Navarra. Pamplona

- Marta Hernández Conde. Hospital Puerta de Hierro-Majadahonda. Madrid

\section{Requisitos necesarios:}

- Coordinador: David Martínez Ares. Complejo Hospitalario Universitario de Vigo. Vigo

- Fernando Alberca de las Parras. Hospital Clínico Universitario Virgen de la Arrixaca. Murcia

- Carla Jerusalén Gargallo Puyuelo. Hospital Clínico Universitario Lozano Blesa. Zaragoza

- Esteban Saperas Franch. Hospital General de Catalunya. Sant Cugat del Vallés, Barcelona 
Apéndice 1 (Cont.). Componentes del panel de expertos encargado de realizar la guía

\section{Información al paciente:}

- Coordinadora: Maite Herráiz Bayod. Clínica Universidad de Navarra. Pamplona

- Miguel Muñoz Navas. Clínica Universidad de Navarra. Pamplona

- Javier Gordillo. Enfermero. Unidad de Endoscopia. Complejo Hospitalario de Navarra. Pamplona

- Felipe Ramos Zabala. Hospital Universitario Montepríncipe. Madrid

- Jose María Echevarría. Paciente de la Alianza PCCR

\section{Identificación y caracterización de las lesiones:}

- Coordinadora: Akiko Ono. Hospital Clínico Universitario Virgen de la Arrixaca. Murcia

- Marco Bustamante. Hospital Universitari i Politècnic La Fe de Valencia. Valencia

- Marco Antonio Álvarez. Hospital del Mar. Barcelona

- Mariano González-Haba. Hospital Puerta de Hierro-Majadahonda. Madrid

- Marta Montes Díaz. Anatomía Patológica. Complejo Hospitalario de Navarra. Pamplona

Aspectos técnicos:

- Coordinador: Andrés Sánchez Yagüe. Hospital Costa del Sol. Marbella, Málaga

- Begoña González-Suárez. Hospital Universitari Clínic. Barcelona

- Juan José Vila Costas. Complejo Hospitalario de Navarra. Pamplona

- Carlos Guarner Argente. Hospital de la Santa Creu i San Pau. Barcelona

- Ferran González-Huix. Hospital Universitari de Girona Doctor Josep Trueta. Girona

\section{Complicaciones:}

- Coordinadora: María Fraile González. Hospital de San Pedro. Logroño

- Fernando Múgica Aguinaga. Hospital Universitario Donostia. Donostia-San Sebastián

- Julyssa Cobián. Hospital Universitario Donostia. Donostia-San Sebastián

- Joaquín Rodríguez Sánchez. Hospital General de Ciudad Real. Ciudad Real

- Bartolome López Viedma. Hospital General de Ciudad Real. Ciudad Real

\section{Eficacia:}

- Coordinador: Pedro Alonso Aguirre. Complejo Hospitalario Universitario de A Coruña. A Coruña

- Noel Pin Vieito. Complejo Hospitalario de Ourense. Ourense

- José Carlos Marín Gabriel. Hospital Universitario 12 de Octubre. Madrid

- Óscar Nogales Rincón. Hospital General Universitario Gregorio Marañón. Madrid

- Eduardo Valdivielso Cortázar. Complejo Hospitalario Universitario de A Coruña. A Coruña

\section{REM, DES o cirugía:}

- Coordinador: Adolfo Parra Blanco. NIHR Nottingham Digestive Diseases Biomedical Research Unit. Nottingham, Reino Unido

- Joaquín de la Peña. Hospital de Valdecilla. Santander

- José Díaz Tasende. Hospital Universitario 12 de Octubre. Madrid

- Francisco Javier Navajas León. Complejo Hospitalario de Toledo. Toledo

- Helena León Brito. Hospital Reina Sofía. Tudela, Navarra

\section{Seguimiento:}

- Coordinador: Santiago Soto Iglesias. Complexo Hospitalario de Ourense. Ourense

- David Remedios Espino. Complexo Hospitalario de Ourense. Ourense

- Jose Miguel Esteban. Hospital Universitario Clínico San Carlos. Madrid

- David Barquero Declara. Hospital Moisès Broggi. Barcelona

\section{Aprendizaje y competencia:}

- Coordinador: Eduardo Redondo Cerezo. Hospital Virgen de las Nieves. Granada

- Juan Gabriel Martínez Cara. Hospital Virgen de las Nieves. Granada

- Felipe Martínez Alcalá. Centro Andaluz de Gastroenterología Integral. Sevilla

- Iñaki Fernández-Urién Sainz. Complejo Hospitalario de Navarra. Pamplona

\section{Conclusiones y líneas futuras a desarrollar:}

- Eduardo Albéniz Arbizu. Complejo Hospitalario de Navarra. Pamplona

- María Pellisé Urquiza. Hospital Universitari Clínic. Barcelona 
Apéndice 2. Recomendaciones generales del Guidelines International Network (adaptado de Qaseem A y cols. Ann Intern Med 2012)

\begin{tabular}{|c|c|c|c|}
\hline \multicolumn{4}{|c|}{ Red internacional de guías: componentes clave para guías de alta calidad y confianza } \\
\hline Componente & Descripción & Sí & No \\
\hline $\begin{array}{l}\text { Composición del grupo que desarrolla } \\
\text { la guía }\end{array}$ & $\begin{array}{l}\text { El grupo de individuos que desarrolla la guía debe incluir: profesionales de la } \\
\text { salud, metodólogos, expertos y pacientes }\end{array}$ & $\mathrm{X}$ & \\
\hline Proceso de toma de decisiones & $\begin{array}{l}\text { La guía debe describir el procedimiento utilizado para alcanzar el consenso } \\
\text { entre el panel de miembros que la elabora y, si es el caso, de la organización } \\
\text { que esponsoriza la guía } \\
\text { Este procedimiento se debe establecer antes de comenzar el desarrollo de la } \\
\text { guía }\end{array}$ & $\mathrm{X}$ & \\
\hline Conflicto de intereses & $\begin{array}{l}\text { La guía debe incluir los conflictos de intereses financieros o no financieros de } \\
\text { todos los miembros que la desarrollan } \\
\text { Además, la guía debe describir cómo se recoge y resuelve cada uno de los } \\
\text { conflictos }\end{array}$ & $\mathrm{X}$ & \\
\hline Ámbito de aplicación de la guía & La guía debe especificar sus objetivos y ámbito de aplicación & $X$ & \\
\hline Métodos & La guía debe describir con detalle los métodos utilizados para su desarrollo & $X$ & \\
\hline Revisión de la evidencia & $\begin{array}{c}\text { El panel de sujetos que elabora la guía debe utilizar métodos para la revisión } \\
\text { sistemática de la evidencia con el fin de identificar y evaluar la evidencia } \\
\text { relacionada con el tema a tratar }\end{array}$ & $\mathrm{X}$ & \\
\hline Recomendaciones de la guía & $\begin{array}{l}\text { Las recomendaciones de la guía deben quedar claramente expuestas } \\
\text { Se deben fundamentar en la evidencia científica de los beneficios, las } \\
\text { contrapartidas y, si es posible, los costes }\end{array}$ & $\mathrm{X}$ & \\
\hline $\begin{array}{l}\text { Clasificación de la evidencia y } \\
\text { recomendaciones }\end{array}$ & $\begin{array}{l}\text { La guía debe usar un sistema de clasificación con el fin de comunicar la } \\
\text { calidad y fiabilidad tanto de la evidencia como de las recomendaciones }\end{array}$ & $X$ & \\
\hline Revisión por pares y revisión externa & $\begin{array}{l}\text { La revisión por expertos no involucrados en el diseño de la guía debe } \\
\text { realizarse antes de ser publicada }\end{array}$ & $\mathrm{X}$ & \\
\hline Caducidad y actualización de la guía & $\begin{array}{l}\text { La guía debe incluir una fecha de caducidad y describir el proceso que se } \\
\qquad \text { Ilevará a cabo para su actualización }\end{array}$ & $\mathrm{X}$ & \\
\hline Financiación y mecenazgo & $\begin{array}{l}\text { Una guía debe revelar toda ayuda para su desarrollo, tanto de la revisión de la } \\
\text { evidencia como de las recomendaciones de la guía }\end{array}$ & $\mathrm{X}$ & \\
\hline
\end{tabular}

Apéndice 3. Scottish Intercollegiate Guidelines Network (SIGN) (adaptado de http://www.sign.ac.uk/methodology)

\begin{tabular}{|c|c|}
\hline \multicolumn{2}{|r|}{ Niveles de evidencia } \\
\hline $1++$ & Metaanálisis de alta calidad, revisiones sistemáticas de ensayos clínicos aleatorizados (ECA) o ECA con muy bajo riesgo de sesgos \\
\hline $1+$ & Metaanálisis metodológicamente bien diseñados, revisiones sistemáticas o ECA con bajo riesgo de sesgos \\
\hline $2++$ & $\begin{array}{l}\text { Revisiones sistemáticas de elevada calidad de estudios casos y controles o cohortes } \\
\text { Estudios de casos y controles o de cohortes de elevada calidad con muy bajo riesgo de sesgos y elevada probabilidad de relación causal }\end{array}$ \\
\hline 2- & $\begin{array}{l}\text { Estudios de casos y controles o de cohortes bien diseñados con elevado riesgo de sesgos y probabilidad significativa de que la } \\
\text { relación no sea causal }\end{array}$ \\
\hline 3 & Estudios no analíticos (por ejemplo, series de casos o casos aislados) \\
\hline 4 & Opinión de expertos \\
\hline
\end{tabular}

\section{Grados de recomendación}

Al menos un metaanálisis, revisión sistemática o ECA clasificado 1++,

A y directamente aplicable a la población diana; 0 un cuerpo de evidencia consistente en estudios clasificados 1+, directamente aplicables a la población diana, y que demuestra consistencia de los resultados 
Apéndice 3 (Cont.). Scottish Intercollegiate Guidelines Network (SIGN) (adaptado de http://www.sign.ac.uk/ methodology)

\begin{tabular}{c|l} 
Grados de recomendación \\
B & $\begin{array}{l}\text { Un cuerpo de evidencia que incluye estudios clasificados } 2++ \text {, directamente aplicable a la población diana y que demuestra } \\
\text { globalmente consistencia de los resultados; o evidencia extrapolable de estudios clasificados } 1++0 \text { 1+ }\end{array}$ \\
C & $\begin{array}{l}\text { Un cuerpo de evidencia que incluye estudios clasificados } 2+\text {, directamente aplicable a la población diana y que demuestra } \\
\text { globalmente consistencia de los resultados; o evidencia extrapolable de estudios clasificados } 2++\end{array}$ \\
D & Nivel de evidencia 3 o 4; o evidencia extrapolable de estudios clasificados $2+$ \\
\hline
\end{tabular}

Apéndice 4. Clasificación de Viena modificada (adaptado de Dixon MF. Gut 2002)

\section{No neoplasia}

- Categoría Viena 1 (negativo para neoplasia)

2. Neoplasia mucosa de bajo grado

- Categoría Viena 3 (neoplasia no invasiva de bajo grado, adenoma de bajo grado, displasia de bajo grado)

- Otras terminologías comunes: displasia leve o moderada

- OMS: neoplasia intraepitelial de bajo grado

3. Neoplasia mucosa de alto grado

- Categoría Viena 4.1-4.4 (neoplasia no invasiva de alto grado, adenoma de alto grado, displasia de alto grado)

- Carcinoma no invasivo (carcinoma in situ)

- Carcinoma intramucoso

- Otra terminología común: displasia de alto grado, displasia severa, neoplasia intraepitelial de alto grado

- OMS: neoplasia intraepitelial de alto grado

- TNM: pTis

4. Carcinoma que invade la submucosa o más en profundidad

- 4a. Carcinoma limitado a la submucosa Categoría Viena 5: (invasión submucosa del carcinoma) TNM: pT1

- 4b. Carcinoma más allá de la submucosa TNM: pT2-T4

1. Para la Clasificación revisada de Viena ver ref. 29.

2. No se recomienda emplear la categoría 2 de la Clasificación de Viena (indefinido) para el cribado.

\section{Apéndice 5. Algoritmo para el manejo de los cánceres precoces colorrectales}

\begin{tabular}{|c|c|c|c|}
\hline \multicolumn{4}{|c|}{ Pólipo resecado endoscópicamente con adenocarcinoma infiltrante } \\
\hline \multicolumn{3}{|c|}{ Resección completa } & \multirow{2}{*}{$\begin{array}{c}\text { Resección incompleta } \\
\text { - En caso de margen profundo } \\
\text { afecto se recomienda CIRUGÍA } \\
\text { - En caso de margen lateral afecto } \\
\text { se puede valorar SEGUIMIENTO }\end{array}$} \\
\hline & & & \\
\hline \multirow{11}{*}{$\begin{array}{l}\text { Bajo riesgo LMN } \\
\text { - Bien/moderadamente } \\
\text { diferenciado } \\
\text { - Sésiles/planos: infiltración sm } \\
\text { igual o menor a } 1 \mathrm{~mm} \\
\text { - Pediculados: Haggitt 1-2 o } \\
\text { invasión sm menor de } 3 \mathrm{~mm} \\
\text { - No invasión linfática/vascular } \\
\text { - Ausencia de Budding } \\
\text { - Margen profundo mayor o } \\
\text { igual a } 1 \text { mm }\end{array}$} & Riesgo medio LMN & Riesgo alto LMN & \\
\hline & - Sésiles/planos: infiltración sm & - Alto grado/pobremente & \\
\hline & entre 1001 y 2000 um & diferenciado & \\
\hline & - Pediculados: Haggitt 3 o invasión & - Sésiles/planos: infiltración sm & \\
\hline & - Buddina de hain arado & _ Pediculados. Hagaitt 4 ninvasión & \\
\hline & - Margen profundo no valorable & sm mayor de $3 \mathrm{~mm}$ & \\
\hline & - Tamaño del CCR menor de 4 mm & - Invasión linfática/vascular & \\
\hline & & - Budding de alto grado & \\
\hline & & - Margen profundo menos de $1 \mathrm{~mm}$ & \\
\hline & & - Tamaño del CCR mayor de 4 mm & \\
\hline & \multicolumn{2}{|c|}{ Comité mutidisciplinar } & \\
\hline Vigilancia & \multicolumn{2}{|c|}{ Vigilancia o cirugía } & \\
\hline
\end{tabular}




\section{BIBLIOGRAFÍA}

1. Oaseem A, Forland F, Macbeth F, et al; Board of Trustees of the Guidelines International Network. Guidelines International Network: Toward International Standards for Clinical Practice Guidelines. Ann Intern Med 2012;156(7):525-31. DOI: 10.7326/0003-4819-156-7-201204030-00009

2. Scottish Intercollegiate Guidelines Network. Methodology. Available at: http://www.sign.ac.uk/methodology.html

3. Puli SR, Kakugawa Y, Saito Y, et al. Successful complete cure en-bloc resection of large nonpedunculated colonic polyps by endoscopic submucosal dissection: A meta-analysis and systematic review. Ann Surg Oncol 2009;16(8):2147-51. DOI: 10.1245/s10434-009-0520-7

4. Masci E, Viale E, Notaristefano $\mathrm{C}$, et al. Endoscopic mucosal resection in high- and low-volume centers: A prospective multicentric study. Surg Endosc 2013;27(10):3799-805. DOl: 10.1007/s00464-013-2977-5

5. Moss A, Bourke MJ, Williams SJ, et al. Endoscopic mucosal resection outcomes and prediction of submucosal cancer from advanced colonic mucosal neoplasia. Gastroenterology 2011;140(7):1909-18. DOI: 10.1053/j. gastro.2011.02.062

6. Moss A, Williams SJ, Hourigan LF, et al. Long-term adenoma recurrence following wide-field endoscopic mucosal resection (WF-EMR) for advanced colonic mucosal neoplasia is infrequent: Results and risk factors in 1000 cases from the Australian Colonic EMR (ACE) study. Gut 2015;64(1):57-65. DOI: 10.1136/gutjnl-2013-305516

7. Marín-Gabriel JC, Díaz-Tasende J, Rodríguez-Muñoz S. Colonic endoscopic full-thickness resection (EFTR) with the over-the-scope device (FTRD): A short case series. Rev Esp Enferm Dig 2017;109(3):230-3. DOI: 10.17235/ reed.2017.4259/2016

8. Wang J, Zhang $\mathrm{X}-\mathrm{H}, \mathrm{Ge} \mathrm{J}$, et al. Endoscopic submucosal dissection vs endoscopic mucosal resection for colorectal tumors: A meta-analysis. World J Gastroenterol 2014;20:8282-7. D0I: 10.3748/wjg.v20.i25.8282

9. Larach SW. Transanal endoscopic microsurgery (TEM) and transanal minimally invasive surgery (TAMIS). Cir Esp 2012;90:418-20. DOI: 10.1016/j. ciresp.2012.04.004

10. Tanaka S, Terasaki M, Hayashi N, et al. Warning for unprincipled colorectal endoscopic mucosal dissection: Accurate diagnosis and reasonable treatment strategy. Dig Endosc 2013;25:107-16. DOI: 10.1111/den.12016

11. Rex DK, Ahnen DJ, Baron JA, et al. Serrated lesions of the colorectum: Review and recommendations from an expert panel. Am J Gastroenterol 2012;107(9):1315-29. DOI: 10.1038/ajg.2012.161

12. Burgess NG, Pellisé M, Nanda KS, et al. Clinical and endoscopic predictors of cytological dysplasia or cancer in a prospective multicentre study of large sessile serrated adenomas/polyps. Gut 2016;65(3):437-46. DOI: 10.1136/gutjnl-2014-308603

13. Pellisé $M$, Burgess NG, Tutticci $N$, et al. Endoscopic mucosal resection for large serrated lesions in comparison with adenomas: A prospective multicentre study of 2000 lesions. Gut 2017;66:644-53. D0I: 10.1136/gutjnl-2015-310249

14. Zhong DD, Shao LM, Cai JT. Endoscopic mucosal resection vs endoscopic submucosal dissection for rectal carcinoid tumours: A systematic review and meta-analysis. Colorectal Dis 2013;15:283-91. DOI: 10.1111/ codi.12069

15. Pérez-Cuadrado Robles E, Yamada M, Saito Y. Succesfull ballon overtube-guided colorectal endoscopic submucosal dissection by a gastroscope. Rev Esp Enferm Dig 2016;108(5):280-1.

16. Igea F, Casellas JA, González-Huix F, et al. Sedación en endoscopia digestiva. Guía de práctica clínica de la Sociedad Española de Endoscopia Digestiva. Rev Esp Enferm Dig 2014;106(3):195-211.

17. American Society of Anesthesiologists Task Force on Preanesthesia Evaluation. Practice advisory for preanesthesia evaluation: A report by the American Society of Anesthesiologists Task Force on Preanesthesia evaluation. Anesthesiology 2002;96:485-96. DOI: 10.1097/00000542200202000-00037
18. American Society of Gastrointestinal Endoscopy; Technology Assessment Committee. Implanted electronic devices at endoscopy: Advice in a gray area. Gastrointest Endosc 2007;65:561-8.

19. Allison MC, Sandoe JA, Tighe R, et al; Endoscopy Committee of the British Society of Gastroenterology. Antibiotic prophylaxis in gastrointestinal endoscopy. Gut 2009;58:869-80. DOI: 10.1136/gut.2007.136580

20. Boustiere C, Veitch A, Vanbiervliet G, et al; European Society of Gastrointestinal Endoscopy. Endoscopy and antiplatelet agents. Endoscopy 2011:43:445-61.

21. Alberca de las Parras F, Marín F, Roldán Schilling V, et al. Manejo de los fármacos antitrombóticos asociado a procedimientos endoscópicos. Rev Esp Enferm Dig 2015;107:289-306.

22. Artículo 5 de la Ley $41 / 2002$, de 14 de noviembre, reguladora de la autonomía del paciente y de derechos y obligaciones en materia de información y documentación clínica.

23. Dolz Abadía C. Consentimiento informado en endoscopia digestiva: información para el paciente, protección para el endoscopista. Rev Esp Enferm Dig 2016;108(Supl. II):13-23.

24. Kaminski MF, Hassan C, Bisschops R, et al. Advanced imaging for detection and differentiation of colorectal neoplasia: European Society of Gastrointestinal Endoscopy (ESGE) Guideline. Endoscopy 2014:46:435-9. DOI: $10.1055 / s-0034-1365348$

25. Pohl J, Schenider A, Vogell H, et al. Pancolonic chromoendoscopy with indigo carmine versus standard colonoscopy for detection of neoplastic lesions: A randomised two-centre trial. Gut 2011;60:485-90. D0I: 10.1136/ gut.2010.229534

26. Endoscopic Classification Review Group. Update on the Paris classification of superficial neoplastic lesions in the digestive tract. Endoscopy 2005;58(6):570-8. DOI: 10.1055/s-2005-861352

27. Hurlstone DP, Sanders DS, Cross SS, et al. Colonoscopic resection of lateral spreading tumours: A prospective analysis of endoscopic mucosal resection. Gut 2004;53(9):1334-9. DOI: 10.1136/gut.2003.036913

28. Ueno H, Mochizuki H, Hashiguchi Y, et al. Risk factors for an adverse outcome in early invasive colorectal carcinoma. Gastroenterology 2004;127:35894. DOI: 10.1053/j.gastro.2004.04.022

29. Kudo S, Hirota S, Nakajima T, et al. Colorectal tumours and pit pattern. J Clin Pathol 1994;47:880-5. DOI: 10.1136/jcp.47.10.880

30. Sano Y, Kobayashi M, Hamamoto $Y$, et al. New diagnostic method based on color imaging using narrow band imaging (NBI) system for gastrointestinal tract. Gastrointest Endosc 2001;53:AB125.

31. Tanaka S, Sano Y. Aim to unify the narrow band imaging (NBI) magnifying classification for colorectal tumors: Current status in Japan from a summary of the consensus symposium in the 79th Annual Meeting of the Japan Gastroenterological Endoscopy Society. Digest Endosc 2011;23:1319. DOI: 10.1111/j.1443-1661.2011.01106.x

32. Quirke P, Risio M, Lambert R, et al; International Agency for Research on Cancer. European guidelines for quality assurance in colorectal cancer screening and diagnosis. First Edition - Quality assurance in pathology in colorectal cancer screening and diagnosis. Endoscopy 2012:44(3):SE11630. DOI: $10.1055 / \mathrm{s}-0032-1309797$

33. Dixon MF. Gastrointestinal epithelial neoplasia: Vienna revisited. Gut 2002:51:130-1. DOI: 10.1136/gut.51.1.130

34. Kobayashi N, Saito $Y$, Sano $Y$, et al. Determining the treatment strategy for colorectal neoplastic lesions: Endoscopic assessment or the non-lifting sign for diagnosing invasion depth? Endoscopy 2007;3988:701-5. DOI: 10.1055/s-2007-966587

35. Monkemüller K. State of the art. Advanced colon polypectomy. Clin Gastroenterol Hepatol 2009;7:641-52. D0I: 10.1016/j.cgh.2009.02.032

36. Tokar JL, Barth BA, Banerjee S, et al; ASGE Technology Committee. Electrosurgical Generators. Gastrointest Endosc 2013;78:197-208. DOI: 10.1016/j.gie.2013.04.164 
37. Uraoka T, Fujii T, Saito $Y$, et al. Effectiveness of glycerol as a submucosal injection for EMR. Gastrointest Endosc 2005;61:736-40. DOI: 10.1016/ S0016-5107(05)00321-4

38. Dolz-Abadía C, Vilella-Martorell A. Submucosal chromoendoscopy. A technique that highlights epithelia and differentiates histological components, and renders colon polypectomy easier and safer. Rev Esp Enferm Dig 2015;107(7):430-5. DOl: 10.17235/reed.2015.3550/2014

39. Sanchez-Yagüe A, Kantelbach T, Raju G, et al. Advanced endoscopic resection of colorectal lesions. Gastroenterol Clin N Am 2013;42:459-77. DOI: 10.1016/j.gtc.2013.05.012

40. Rex DK. Two-step treatment of lateral spreading tumors. Gastrointest Endosc 2010;72:468. DOI: 10.1016/j.gie.2009.11.032

41. Binmoeller KF, Weilert F, Shah J, et al. Underwater EMR without submucosal injection for large sessile colorectal polyps (with video). Gastrointest Endosc 2012;75:1086-91. DOI: 10.1016/j.gie.2011.12.022

42. Sanchez-Yagüe A, Yamaguchi Y, Takao T, et al. Endoscopic mucosal dissection of a lower rectal polyp proximal to the dentate line by using local lidocaine injection. Gastrointest Endosc 2011;73:406-7. DOI: 10.1016/j. gie.2010.06.044

43. Kim HS, Kim TI, Kim WH, et al. Risk factors for immediate postpolypectomy bleeding of the colon: A multicenter study. Am J Gastroenterol 2006;101:1333-41. DOI: 10.1111/j.1572-0241.2006.00638.x

44. Fu KI, Fujii T, Kato S, et al. A new endoscopic tattooing technique for identifying the location of colonic lesions during laparoscopic surgery: A comparison with the conventional technique. Endoscopy 201;33:687-91.

45. Burgess NG, Metz AJ, Williams SJ, et al. Risk factors for intraprocedural and clinically significant delayed bleeding after wide-field endoscopic mucosal resection of large colonic lesions. Clin Gastroenterol Hepatol 2014;12:651-61. DOI: 10.1016/j.cgh.2013.09.049

46. Albéniz E, Fraile $M$, Ibáñez $B$, et al. A scoring system to determine risk of delayed bleeding after endoscopic mucosal resection of large colorectal lesions. Clin Gastroenterol Hepatol 2016;14:1140-7. DOl: 10.1016/j. cgh.2016.03.021

47. Albéniz E, Fraile M, Martínez-Ares D, et al. Complications after endoscopic mucosal resection of large colorectal lesions: A multicenter Spanish EMR Group Study. United European Gastroenterol J 2014;2:A283.

48. Dobrowolski S, Dobosz M, Babicki A, et al. Prophylactic submucosal saline-adrenaline injection in colonoscopic polypectomy: Prospective randomized study. Surg Endosc 2004;18:990-3. DOI: 10.1007/s00464-003-9214-6

49. Bahin FF, Naidoo M, Williams SJ, et al. Prophylactic endoscopic coagulation to prevent bleeding after wide-field endoscopic mucosal resection of large sessile colorectal polyps. Clin Gastroenterol Hepatol 2015;13:724-30. DOl: 10.1016/j.cgh.2014.07.063
50. Panteris V, Haringsma K, Kuipers EJ. Colonoscopy perforation rate, mechanisms and outcome: From diagnostic to therapeutic colonoscopy. Endoscopy 2009;41:941-51. DOI: 10.1055/s-0029-1215179

51. Heresbach D, Kornhauser R, Seyrig JA, et al. Omega Group. A national survey of endoscopic mucosal resection for superficial gastrointestinal neoplasia. Endoscopy 2010;42:806-13. DOI: 10.1055/s-0030-1255715

52. Taku K, Sano Y, Fu Kl, et al. latrogenic perforation associated with therapeutic colonoscopy: A multicenter study in Japan. J Gastroenterol Hepatol 2007;22:1409-14. DOI: 10.1111/j.1440-1746.2007.05022.x

53. Bielawska B, Day AG, Lieberman DA, et al. Risk factors for early colonoscopic perforation include non-gastrointestinal endoscopists: A multivariable analysis. Clin Gastroenterol Hepatol 2014;12:85-92. DOI: 10.1016/j. cgh.2013.06.030

54. Paspatis GA, Dumonceau JM, Barthet $\mathrm{M}$, et al. Diagnosis and management of iatrogenic endoscopic perforations: European Society of Gastrointestinal Endoscopy (ESGE) Position Statement. Endoscopy 2014;46:693711. DOI: $10.1055 / \mathrm{s}-0034-1377531$

55. Belderbos T, Leenders M, Moons L, et al. Local recurrence after endoscopic mucosal resection of nonpedunculated colorectal lesions: Systematic review and meta-analysis. Endoscopy 2014;46:388-400. DOI: 10.1055/s0034-1364970

56. Hassan C, Repici A, Sharma P, et al. Efficacy and safety of endoscopic resection of large colorectal polyps: A systematic review and meta-analysis. Gut 2015;0:1-15.

57. Knabe M, Pohl J, Gerges C, et al. Standarized long-term floow-up after endoscopic resection of large nonpedunculated colorectal lesions: A prospective two-center study. Am J Gastroenterol 2014;109:183-9. DOI: 10.1038/ajg.2013.419

58. Leung K, Pinsky P, Laiyemo A0, et al. Ongoing colorectal cancer risk despite surveillance colonoscopy: The Polyp Prevention Trial Continued Follow-Up Study. Gastrointest Endosc 2010;71:111-7. DOI: 10.1016/j.gie.2009.05.010

59. Hassan C, Quintero E, Dumonceau JM, et al. Post-polypectomy colonoscopy surveillance: European Society of Gastrointestinal Endoscopy (ESGE) Guideline. Endoscopy 2013;45:842-51. DOI: 10.1055/s-0033-1344548

60. Rembacken B, Hassan C, Riemann JF, et al. Quality in screening endoscopy: Position statement of European Society of Gastrointestinal Endoscopy (ESGE). Endoscopy 2012:44(10):957-68. D0I: 10.1055/s-0032-1325686

61. Brooker JC, Saunders BP, Shah SG, et al. Endoscopic resection of large sessile colonic polyps by specialist and non-specialist endoscopists. $\mathrm{Br} \mathrm{J}$ Surg 2002;89:1020-4. DOI: 10.1046/j.1365-2168.2002.02157.x

62. Endoscopy trainees. Joint Advisory Group on GI Endoscopy (JAG). Royal College of Physicians. Available at: https://www.thejag.org.uk/CMS/ Page. aspx?Pageld=65 